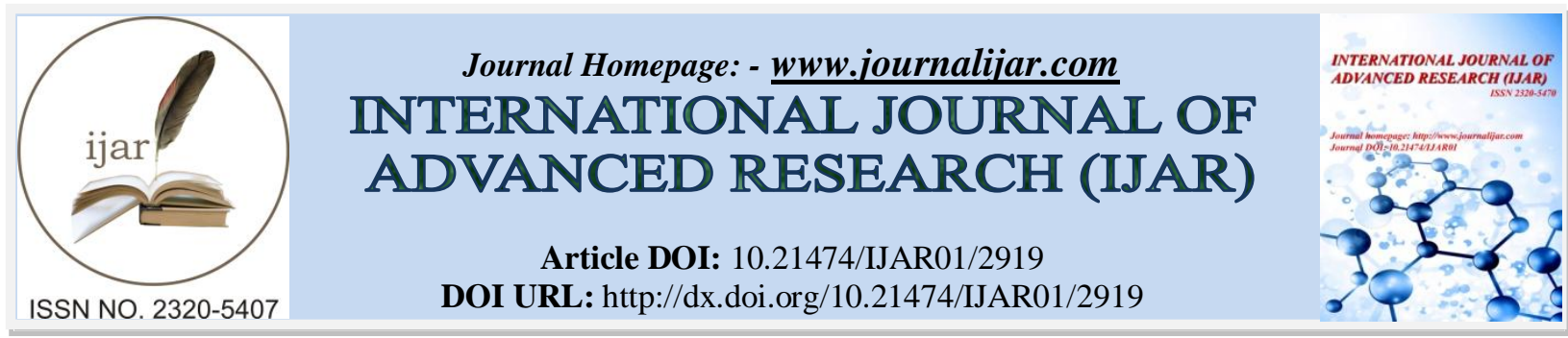

RESEARCH ARTICLE

\title{
HELICOBACTER PYLORI ERADICATION EFFECT ON LIVER FAT CONTENT IN PATIENT WITH NON-ALCOHOLIC FATTY LIVER DISEASE.
}

Ahmed H. Arnaout.
Lecturer of internal medicine, faculty of medicine, Zagazig University.

\section{Manuscript Info}

Manuscript History

Received: 24 November 2016

Final Accepted: 25 December 2016

Published: January 2017

Key words:-

Helicobacter pylori, Non-alcoholic Fatty

Liver Disease, Insulin Resistance,

Alanine aminotransferase, aspartate

aminotransferase.

\section{Abstract}

Background: Helicobacter pylori (HP) infection role in nonalcoholic fatty liver disease (NAFLD) pathogenesis is controversial.

Aims: This study aimed to evaluate the effect of HP eradication on liver fat content (LFC), liver function tests (LFT), lipid profile, and homeostasis model assessment-IR (HOMA-IR) index in NAFLD.

Patients and Methods: Patient with dyspepsia and increased serum levels of aminotransferases enrolled in the study. Exclusion criteria were factors affecting aminotransferases levels or HP treatment regimen. Patients with elevated aminotransferases levels and ultrasound findings of fatty liver disease were supposed for NAFLD. NAFLD liver fat score used to classify NAFLD, those with score greater than -0.64 and positive results for urea breath test (UBT), were included. Patients underwent lifestyle modifications and HP eradication. Alanine aminotransferase (ALT), aspartate aminotransferase (AST), alkaline phosphatase (ALP), triglyceride (TG), cholesterol (CHOL), high and low-density lipoprotein (HDL, LDL), fasting serum glucose (FSG), LFC, and HOMA-IR were checked at baseline, after eight weeks and twenty four weeks.

Results : One hundred twenty patients (58 males) with the mean age of 45.39 ( \pm 9.24$)$ were included with repeated ANOVA measurement showed a significant reduction in anthropometric measurements, laboratory parameters (except for HDL) and LFC in both groups during the study; however, no significant difference was observed between the groups.

Conclusions: HP eradication per se might not affect LFT, lipid profile, LFC, and insulin resistance in dyspeptic NAFLD patients

Copy Right, IJAR, 2016,. All rights reserved.

\section{Introduction:-}

Recently the relation between the gut microbes and the development and progression of liver cell damage has come to interest, regarding to this, helicobacter pylori (HP) deoxy-ribonucleic acid (DNA) was detected in patients with different causes of chronic liver disease (CLD) including non-alcoholic fatty liver disease (NAFLD) (1-4). Regardless of HP DNA existence in liver samples, no bacteria could be cultured (5).

Corresponding Author:- Ahmed H. Arnaout.

Address:- lecturer of internal medicine, faculty of medicine, Zagazig university. 
Different theories for the mechanisms of the NAFLD development in patients with HP infection were issued, one proposed that HP species would produce liver specific toxin causes liver cell damage (6), another one suggested that HP invasion to intestinal mucosa might increase gut permeability and allow the bacterial endotoxin to pass via the portal vein to the liver (7). Because HP eradication is not such difficult in most cases, discovering its role in diseases apart from the stomach could be of great importance for public health, currently the way in which HP eradication would affect clinical state of patients with NAFLD is not well cleared (8-11).

\section{Aim of the study:-}

The aim of this study was to determine the short term effect of HP eradication on liver fat content, LFTs, and metabolic profile (biochemical parameters and anthropometric measurements) in a sample of dyspeptic NAFLD patients.

\section{Patients and Methods:-}

This study was done as a randomized clinical trial, each of the patients was assigned to treatment or control group, Participants signed a written consent prior to enrollment.

Adult patients referred to the gastroenterology clinic of a general hospital due to increased serum aminotransferase levels were selected. Serum alanine aminotransferase (ALT) and aspartate aminotransferase (AST) levels $\geq 40$ units per liter (U/L) considered elevated (12). Patients with dyspepsia, positive results for anti HP antibody and ultrasonographic evidence of fatty liver were enrolled; the exclusion criteria were factors affecting serum aminotransferase or HP treatment strategy (13). All patients with elevated serum aminotransferase levels in the first blood sampling were rechecked after two months, and persistently elevated serum aminotransferase levels with ultrasonographic criteria for fatty liver supposed to have NAFLD (13). Considering the liver biopsy limitations, 'NAFLD liver fat score' used in enrolled patients to define NAFLD (14), those with NAFLD liver fat score greater than -0.64 , and positive results for urea breath test (UBT) were included in the study.

Lifestyle modifications was provided to all participants and it consisted of providing calorie-restricted diets and programmed physical activity to achieve ideal body weight based on the guidelines for the diagnosis and management of NAFLD : update 2010 (15) with avoiding of rapid weight loss as it could worsen NAFLD (16), A dietitian checked the patients and controlled their calorie intake.

The patients in treatment group received quadruple treatment with omeprazole (20 mg twice daily), amoxicillin ( $1 \mathrm{~g}$ twice daily), bismuth subcitrate (240 mg twice daily), and clarithromycin (500 mg twice daily) for two weeks (17), the patients requested to bring used packages at the follow up visits weekly, evaluated for compliance and any potential side effects.

The percent of the liver fat content calculated based on the validated formula (14), A radiologist did the liver ultrasonography, the echogenicity of the right lobe of the liver compared to the right kidney with a 3.5 megahertz probe to identify fatty liver (18).

HP antibody (IgG) measured using a quantitative enzyme-linked immunosorbent assay technique, UBT performed by Helioprobe test kit to detect HP infection and eradication. Eradication investigated six weeks after the end of treatment (19). Waist circumference (WC) measured at midpoint between the lower border of rib cage and the top of iliac crest at the end of expiration (20). After a twelve-hour overnight fast, sera of the patients were tested for fasting serum glucose (FSG), ALT, AST, alkaline phosphatase (ALP), triglyceride (TG), cholesterol (CHOL), low-density lipoprotein (LDL) by enzymatic methods (13). Serum insulin concentrations measured based on an enzyme immunoassay. Quantitative measurement of insulin resistance (IR) was done by homeostasis model assessment-IR (HOMA-IR =fasting serum insulin $\times$ fasting serum glucose/22.5) (21). Diabetes mellitus (DM) was diagnosed either previous diagnosis of DM or if FBS equal or greater than $126 \mathrm{mg} / \mathrm{dl}$ after double check.

Primary outcome measure was change from baseline fat content at 6 months. Secondary outcome measures were changes from baseline LFT, metabolic parameters, and anthropometric measurements at 6 months. 
A statistical power analysis used to calculate the sample size. Considering the two-sided level $(\alpha)$ of 0.05 and the power of $90 \%(\beta=0.1)$, a total sample size of 46 patients was determined to detect one percent inter-group difference in liver fat content. 60 patients were enrolled in each treatment group of the study.

Data was summarized as means \pm SD for continuous and number (percentage) for qualitative variables. $T$ test and Chi-square used to compare the mean values of continuous and categorical variable between the treatment groups. Repeated measure analysis of variance (ANOVA) was applied for comparing treatment groups regarding the changes of variables during the study. All statistical analyses were performed using SPSS version 17 (SPSS, Chicago, IL, The USA). The probability of the difference between the dependent and the independent variables were considered significant if a two-tailed $\mathrm{P}$ value was less than 0.05 .

\section{Results:-}

One hundred and forty five patients of suspected NAFLD were evaluated from April 2013 to April 2014. One hundred and twenty patients, with the mean age of $45.35 \pm 9.24$ years, were included in the study. Table 1 represents patient characteristics. Reasons for leaving out were patient unwillingness to participate in the study $(\mathrm{n}=10)$, normalization of ALT during the lead-in phase $(n=14)$, and pregnancy or lactation $(n=1)$.

Table 1:- The baseline characteristics of participants

\begin{tabular}{|c|c|c|c|}
\hline & $\begin{array}{c}\text { Lifestyle Modification plus Helicobacter } \\
\text { pylori eradication }\end{array}$ & Lifestyle Modification & P value \\
\hline Age, mean $\mathbf{E}$ SD, $\mathbf{y}$ & $45.6 \pm 7.3$ & $45.2 \pm 7.1$ & 0.7 \\
\hline Gender & & & 0.5 \\
\hline Male & 26 & 32 & \\
\hline Female & 32 & 28 & 0.5 \\
\hline Diabetes mellitus & & & \\
\hline Present & 26 & 25 & \\
\hline Absent & 32 & 35 & \\
\hline
\end{tabular}

There were no medications side effects occurred requiring dose reduction or discontinuation of the treatment during the study. Good adherence to the therapy was followed by pill counting during the follow up visits. Based on UBT results, the eradication rate was $96 \%$.

All the continuous variables were normally distributed at the baseline measurement. Table 2 shows the comparisons of LFC, anthropometric measurements, and laboratory levels according to the treatment groups during the study. There was no significant difference between the treatment groups at baseline, eight weeks, and twenty four weeks.

Table 2:- The comparison of liver fat content, anthropometric measurements, and laboratory levels between the treatment groups during the study

\begin{tabular}{|l|l|l|l|}
\hline & $\begin{array}{c}\text { Lifestyle Modification plus } \\
\text { Helicobacter pylori eradication }\end{array}$ & Lifestyle Modification & P value \\
\hline Liver fat content, mean $\mathbf{\text { SD }}$ & & & \\
\hline Baseline & $11.9 \pm 6.3$ & $11.6 \pm 5.8$ & 0.7 \\
\hline Eight weeks & $10.5 \pm 5.9$ & $10.1 \pm 5.7$ & 0.8 \\
\hline Twenty four weeks & $9.1 \pm 6.3$ & $8.7 \pm 5.1$ & 0.6 \\
\hline $\begin{array}{l}\text { Waist circumference, mean } \\
\text { SD, cm }\end{array}$ & & & \\
\hline Baseline & $103.2 \pm 1.9$ & $102.1 \pm 2.1$ & 0.4 \\
\hline Eight weeks & $102.8 \pm 2.1$ & $101.9 \pm 2.5$ & 0.3 \\
\hline Twenty four weeks & $99.3 \pm 2.7$ & 100.1 & 0.7 \\
\hline Weight, kg & & & \\
\hline Baseline & $88.3 \pm 9.1$ & $89.2 \pm 7.6$ & 0.6 \\
\hline Eight weeks & $87.5 \pm 10.7$ & $88.1 \pm 6.3$ & 0.7 \\
\hline Twenty four weeks & $80.7 \pm 8.4$ & $80.9 \pm 8.3$ & \\
\hline Body mass index, $\mathbf{k g} / \mathbf{m}^{\mathbf{2}}$ & & & 0.3 \\
\hline Baseline & $32.4 \pm 3.2$ & $31.3 \pm 3.5$ & 0.2 \\
\hline Eight weeks & $31.8 \pm 4.2$ & $30.9 \pm 4.1$ & \\
\hline
\end{tabular}




\begin{tabular}{|c|c|c|c|}
\hline Twenty four weeks & $29.2 \pm 3.1$ & $28.4 \pm 3.3$ & 0.2 \\
\hline \multicolumn{4}{|c|}{$\begin{array}{l}\text { Aspartate aminotransferase, } \\
\text { U/L }\end{array}$} \\
\hline Baseline & $45.9 \pm 18.6$ & $45.7 \pm 18.4$ & 0.8 \\
\hline Eight weeks & $37.8 \pm 15.2$ & $38.3 \pm 17.9$ & 0.7 \\
\hline Twenty four weeks & $32.3 \pm 13.1$ & $33.1 \pm 15.4$ & 0.6 \\
\hline \multicolumn{4}{|c|}{ Alanine aminotransferase, U/L } \\
\hline Baseline & $67.8 \pm 25.4$ & $72.4 \pm 26.4$ & 0.5 \\
\hline Eight weeks & $53.5 \pm 31.1$ & $53.1 \pm 32.5$ & 0.8 \\
\hline Twenty four weeks & $42.2 \pm 23.3$ & $41.7 \pm 24.9$ & 0.8 \\
\hline \multicolumn{4}{|c|}{ Alkaline phosphatase, U/L } \\
\hline Baseline & $181.4 \pm 57.3$ & $189.7 \pm 49.8$ & 0.3 \\
\hline Eight weeks & $171.7 \pm 50.9$ & $186.3 \pm 47.6$ & 0.2 \\
\hline Twenty four weeks & $161.5 \pm 42.1$ & $175.4 \pm 41.6$ & 0.1 \\
\hline \multicolumn{4}{|l|}{ Triglycerides, mg/dl } \\
\hline Baseline & $175.6 \pm 99.7$ & $156.7 \pm 87.9$ & 0.3 \\
\hline Eight weeks & $139.4 \pm 75.3$ & $128.5 \pm 54.9$ & 0.2 \\
\hline Twenty four weeks & $121.5 \pm 47.3$ & $118.3 \pm 42.1$ & 0.5 \\
\hline \multicolumn{4}{|l|}{ Cholesterol, mg/dl } \\
\hline Baseline & $181.7 \pm 30.6$ & $183.4 \pm 39.5$ & 0.6 \\
\hline Eight weeks & $172.8 \pm 28.6$ & $174.2 \pm 32.4$ & 0.7 \\
\hline Twenty four weeks & $168.4 \pm 22.7$ & $169.3 \pm 24.2$ & 0.8 \\
\hline \multicolumn{4}{|c|}{ Low density lipoprotein, mg/dl } \\
\hline Baseline & $102.5 \pm 24.5$ & $109.2 \pm 34.2$ & 0.2 \\
\hline Eight weeks & $98.1 \pm 24.1$ & $103.7 \pm 28.5$ & 0.3 \\
\hline Twenty four weeks & $92.4 \pm 18.7$ & $95.8 \pm 22.1$ & 0.5 \\
\hline \multicolumn{4}{|c|}{ High density lipoprotein, mg/dl } \\
\hline Baseline & $44.6 \pm 6.1$ & $43.2 \pm 6.0$ & 0.2 \\
\hline Eight weeks & $46.1 \pm 5.9$ & $45.8 \pm 5.9$ & 0.5 \\
\hline Twenty four weeks & $51.6 \pm 6.8$ & $49.8 \pm 7.9$ & 0.6 \\
\hline \multicolumn{4}{|c|}{ Fasting plasma glucose, $\mathrm{mmol} / \mathrm{L}$} \\
\hline Baseline & $5.9 \pm 0.7$ & $5.8 \pm 0.6$ & 0.5 \\
\hline Eight weeks & $5.5 \pm 0.4$ & $5.5 \pm 0.5$ & 0.6 \\
\hline Twenty four weeks & $5.2 \pm 0.6$ & $5.3 \pm 0.5$ & 0.5 \\
\hline \multicolumn{4}{|l|}{ HOMA-IR $^{\mathrm{a}}$} \\
\hline Baseline & $5.2 \pm 1.9$ & $5.0 \pm 2.2$ & 0.6 \\
\hline Eight weeks & $4.2 \pm 1.3$ & $4.2 \pm 1.8$ & 0.5 \\
\hline Twenty four weeks & $3.6 \pm 1.2$ & $3.5 \pm 1.3$ & 0.6 \\
\hline
\end{tabular}

${ }^{a}$ HOMA-IR : homeostasis model assessment - insulin resistance

Table 3 shows the results of repeated measure ANOVA with Greenhouse-Geisser correction that determined that the mean studied parameters differed statistically significantly between the time points. Post hoc tests using the Bonferroni correction revealed that all treatment groups showed statistically significant reduction in LFC, anthropometric measurements, and laboratory values (except for HDL) from baseline to the end of the study.

Table 3:- The Comparison of Liver Fat Content, Anthropometric Measurements and Laboratory Parameters Change regarding the treatment Groups during the Study ${ }^{\mathrm{a}}$

\begin{tabular}{|l|c|c|}
\hline \multicolumn{2}{|c|}{ Pair Wise Comparison Between Baseline and the End of treatment } \\
\hline & Mean \pm SD & P value \\
\hline Liver fat content, \% & $3.4 \pm 0.2$ & $<0.01$ \\
\hline Waist circumference, $\mathbf{~ c m}$ & $2.6 \pm 0.3$ & $<0.01$ \\
\hline Weight, $\mathbf{~ k g}$ & $8.1 \pm 0.2$ & $<0.01$ \\
\hline Body mass index, $\mathbf{~ k g} / \mathbf{m}^{2}$ & $2.8 \pm 0.2$ & $<0.01$ \\
\hline Aspartate aminotransferase, U/L & $12.9 \pm 1.2$ & $<0.01$ \\
\hline
\end{tabular}




\begin{tabular}{|l|c|c|}
\hline Alanine aminotransferase, U/L & $28.3 \pm 2.0$ & $<0.01$ \\
\hline Alkaline phosphatase, U/L & $16.8 \pm 2.5$ & $<0.01$ \\
\hline Triglycerides, mg/dl & $46.3 \pm 5.9$ & $<0.01$ \\
\hline Cholesterol, mg/dl & $14.6 \pm 2.3$ & $<0.01$ \\
\hline Low density lipoprotein, mg/dl & $12.6 \pm 1.7$ & $<0.01$ \\
\hline High density lipoprotein, mg/dl & $-7.2 \pm 0.5$ & $<0.01$ \\
\hline Fasting plasma glucose, mmol/L & $0.7 \pm 0.1$ & $<0.01$ \\
\hline Fasting serum insulin, mU/L & $3.7 \pm 0.4$ & $<0.01$ \\
\hline HOMA-IR $^{\mathrm{b}}$ & $1.5 \pm 0.1$ & \\
\hline
\end{tabular}

${ }^{a}$ Negative values represent the increase of the parameter at that interval

${ }^{\mathrm{b}}$ Abbreviation : HOMA-IR, Homeostasis model assessment-insulin resistance

No statistically significant difference in the changes of mean LFC, laboratory values, and anthropometric measurements between the treatment groups at the study intervals (All $\mathrm{P}$ values $>0.05$ in between-subject effects model).

\section{Discussion:-}

This study was designed to evaluate the effect of HP eradication on LFC, LFT, and metabolic parameters in NAFLD patients. In this study the result of HP eradication per se might not affect LFC, LFT, lipid profile, insulin resistance, and anthropometric patients. As liver ultrasonography is to some extent limited for diagnosis and grading the NAFLD severity (18), however its availability and cost effectiveness makes it an appropriate tool for fatty liver screening and it was used to evaluate fatty liver in patients with persistently elevated liver enzymes level. It was done by a single radiologist to avoid inter-observer variability. Considering the possible complications and poor patient acceptance, "NAFLD liver fat score" was used instead of liver biopsy for NAFLD diagnosis. Sensitivity and specificity of the values greater than -0.64 are $86 \%$ and $71 \%$ respectively, to predict NAFLD (14).

To increase the feasibility and reproducibility of the study, a valid formula was applied to measure liver fat content. A previous study showed the validity of this equation to predict liver fat content considering proton magnetic resonance spectroscopy (PMRS) as the gold standard (14). There was a significant high correlation between liver fat content identified by PMRS and liver fat content calculated by the above formula $(14)(\mathrm{r}=0.7, \mathrm{P}<0.0001)$.

The role of HP in extragastric diseases has come to interest. There is large body evidence showing the association of HP and CLD (22-26). The prevalence of HP like DNA in the liver tissue samples of patients with CLD was significantly higher than the patients with metastatic adenocarcinoma (2). In the study of Ponzetto et al. the prevalence of HP antibody ( $\operatorname{IgG}$ ) was higher in males with HCV associated cirrhosis compared to age-matched male blood donors (23). The genomic sequences corresponding to HP were determined in the liver tissues of patients with $\mathrm{HCC}$ and cirrhosis. It was proposed that HP might be implicated in the progression of cirrhosis in patients with $\mathrm{HCV}$ infection (24).

Specific studies regarding HP and NAFLD are increasing. The presence of HP DNA in one sample of liver tissue from a NAFLD patient was a novel finding in 2008 (3). Aller et al. showed that probiotics might decrease markers of lipid peroxidation and improve liver function tests (LFT) in NAFLD (27). Solga et al. reported that probiotics protect gut epithelial cells from the adhesion and invasion of HP (28). Therefore, it can be concluded that probiotics induce their therapeutic effects in NAFLD via avoidance of intestinal mucosa destruction by HP. It was suggested that HP infection was one of the risk factors for the development of NAFLD (25). The study by Polyzos et al. compared biopsy proven NAFLD patients with matched healthy controls (26). The anti HP antibody (IgG) was higher in NAFLD than controls. This study recommended that HP infection eradication might have therapeutic perspectives in NAFLD treatment.

Lifestyle modifications to control daily calorie intake is considered as the gold standard care in NAFLD patients. This strategy was applied to all participants. Obtaining the ideal body weight resulted in sustained improvement of biochemical (serum liver enzymes and insulin level) and histological findings in NAFLD (29). This is in agreement with the results of our study that showed the significant reduction of LFC, LFT, and metabolic indices (FSG, lipid profile, HOMA-IR, and anthropometric measurements) in both treatment groups during the study. In our study, changes in LFC and LFT were not significantly different in HP eradicated group from controls who did not receive 
eradication treatment. This finding is somehow in accordance with the result of Stalke et al. (1). They reported no correlation between LFT and identification of HP DNA in the liver biopsy samples of CLD patients.

In our study, HP eradication had no additional effect on the metabolic indices changes. It was suggested that gut microbiota might regulate IR (30). Currently, there are controversial results about the effect of HP on insulin resistance. The positive correlation of HP with the metabolic syndrome and the inverse correlation with morbid obesity were reported (8-11). Gunji et al. studied a large sample of Japanese general population (8). HP seropositivity was higher in those with metabolic syndrome than the controls. They suggested that HP infection could be associated with metabolic syndrome. On the other hand, there are reports showing the enhanced risk of obesity following HP cure $(10,11)$. These studies proposed that increased Ghrelin following HP treatment improves the appetite and leads to weight gain. The controversial results of the above studies could be due to the difference in the duration of HP infection, and the type of gastritis in the studied populations.

The limitations of the study and Recommendations, as there is not enough evidence to show the benefit of HP eradication in all infected patients, only dyspeptic NAFLD patients were included in this study. Therefore, the results cannot be generalized to all NAFLD patients. Another limitation of the study was the lack of performing liver biopsy to define the histologic response. Comparing the effect of HP eradication on biochemical and histological changes in NAFLD patients (including non-dyspeptic) with longer follow up duration is recommended.

\section{Summary:-}

This randomized clinical trial was performed on one hundred and twenty dyspeptic NAFLD patients who were randomly assigned to lifestyle modification alone or lifestyle modification plus HP eradication treatment. A dietitian checked the daily calorie intake. HP eradication treatment was performed by standard quadruple therapy for 2 weeks. HP eradication rate was $96 \%$ in this sample of NAFLD patients. A comparison was performed between successful eradication group, and those who did not receive HP eradication. Repeated measure ANOVA showed a significant reduction in LFC, anthropometric measurements, and laboratory parameters (except for HDL) in both groups during the study; however, no significant difference was observed between the two groups.

Within the limitations of this study, it can be concluded that HP eradication in dyspeptic NAFLD patients did not provide any additional improvement in LFC, LFT, FSG, lipid profile, IR, and anthropometric measurements compared to lifestyle modification alone.

\section{References:-}

1. Stalke P, Al-Soud WA, Bielawski KP, Bakowska A, Trocha H, Stepinski J, et al. Detection of Helicobacter species in liver and stomach tissues of patients with chronic liver diseases using polymerase chain reactiondenaturing gradient gel electrophoresis and immunohistochemistry. Scand J Gastroenterol. 2005; 40(9):103241.

2. Pirouz T, Zounubi L, Keivani H, Rakhshani N, Hormazdi M. Detection of Helicobacter pylori in paraffinembedded specimens from patients with chronic liver diseases, using the amplification method. Dig Dis Sci. 2009; 54(7):1456-9.

3. Cindoruk M, Cirak MY, Unal S, Karakan T, Erkan G, Engin D, et al. Identification of Helicobacter species by $16 \mathrm{~S}$ rDNA PCR and sequence analysis in human liver samples from patients with various etiologies of benign liver diseases. Eur J Gastroenterol Hepatol. 2008; 20(1):33-6.

4. Huang Y, Fan XG, Wang ZM, Zhou JH, Tian XF, Li N. Identification of helicobacter species in human liver samples from patients with primary hepatocellular carcinoma. J Clin Pathol. 2004; 57(12):1273-7.

5. Pellicano R, Menard A, Rizzetto M, Megraud F. Helicobacter species and liver diseases: association or causation? Lancet Infect Dis. 2008; 8(4):254-60.

6. Taylor NS, Fox JG, Yan L. In-vitro hepatotoxic factor in Helicobacter hepaticus, H. pylori and other Helicobacter species. J Med Microbiol. 1995; 42(1):48-52.

7. Fukuda Y, Bamba H, Okui M, Tamura K, Tanida N, Satomi M, et al. Helicobacter pylori infection increases mucosal permeability of the stomach and intestine. Digestion. 2001; 63 Suppl 1:93-6.

8. Gunji T, Matsuhashi N, Sato H, Fujibayashi K, Okumura M, Sasabe N, et al. Helicobacter pylori infection is significantly associated with metabolic syndrome in the Japanese population. Am J Gastroenterol. 2008; 103(12):3005-10. 
9. Gunji T, Matsuhashi N, Sato H, Fujibayashi K, Okumura M, Sasabe N, et al. Helicobacter pylori infection significantly increases insulin resistance in the asymptomatic Japanese population. Helicobacter. 2009; 14(5):144-50.

10. Nwokolo CU, Freshwater DA, O'Hare P, Randeva HS. Plasma ghrelin following cure of Helicobacter pylori. Gut. 2003; 52(5):637-40.

11. Wu MS, Lee WJ, Wang HH, Huang SP, Lin JT. A case-control study of association of Helicobacter pylori infection with morbid obesity in Taiwan. Arch Intern Med. 2005; 165(13):1552-5.

12. Pratt DS, Kaplan MM. Evaluation of abnormal liver-enzyme results in asymptomatic patients. N Engl J Med. 2000; 342(17):1266-1271.

13. Krier M, Ahmed A. The asymptomatic outpatient with abnormal liver function tests. Clin Liver Dis. 2009; 13(2):167-177.

14. Kotronen A, Peltonen M, Hakkarainen A, Sevastianova K, Bergholm R, Johansson LM, et al. Prediction of nonalcoholic fatty liver disease and liver fat using metabolic and genetic factors. Gastroenterology. 2009; 137(3):865-72.

15. Fan JG, Jia JD, Li YM, Wang BY, Lu LG, Shi JP, et al. Guidelines for the diagnosis and management of nonalcoholic fatty liver disease: update 2010: (published in Chinese on Chinese Journal of Hepatology 2010; 18:163-166). J Dig Dis. 2011; 12(1):38-44.

16. Suzuki A, Binks M, Sha R, Wachholtz A, Eisenson H, Diehl AM. Serum aminotransferase changes with significant weight loss: sex and age effects. Metabolism. 2010; 59(2):177-85.

17. Fakheri H, Malekzadeh R, Merat S, Khatibian M, Fazel A, Alizadeh BZ, et al. Clarithromycin vs. furazolidone in quadruple therapy regimens for the treatment of Helicobacter pylori in a population with a high metronidazole resistance rate. Aliment Pharmacol Ther. 2001; 15(3):411-6.

18. Razavizade M, Jamali R, Arj A, Talari H. Serum parameters predict the severity of ultrasonographic findings in non-alcoholic fatty liver disease. Hepatobiliary Pancreat Dis Int. 2012; 11(5):513-20.

19. Jonaitis LV, Kiudelis G, Kupcinskas L. Evaluation of a novel 14C-urea breath test "Heliprobe" in diagnosis of Helicobacter pylori infection. Medicina (Kaunas). 2007; 43(1):32-5.

20. Romero-Corral A, Somers VK, Sierra-Johnson J, Thomas RJ, Collazo- Clavell ML, Korinek J, et al. Accuracy of body mass index in diagnosing obesity in the adult general population. Int J Obes (Lond) 2008; 32:959-66.

21. Wallace TM, Levy JC, Matthews DR. Use and abuse of HOMA modeling. Diabetes Care. 2004; 27(6):148795.

22. Dogan Z, Filik L, Ergul B, Sarikaya M, Akbal E. Association between Helicobacter pylori and liver-to-spleen ratio: a randomized-controlled single-blind study. Eur J Gastroenterol Hepatol. 2013; 25(1):107-10.

23. Ponzetto A, Pellicano R, Leone N, Cutufia MA, Turrini F, Grigioni WF, et al. Helicobacter infection and cirrhosis in hepatitis $\mathrm{C}$ virus carriage: is it an innocent bystander or a troublemaker? Med Hypotheses. 2000; 54(2):275-7.

24. Leone N, Pellicano R, Brunello F, Cutufia MA, Berrutti M, Fagoonee S, et al. Helicobacter pylori seroprevalence in patients with cirrhosis of the liver and hepatocellular carcinoma. Cancer Detect Prev. 2003; 27(6):494-7.

25. Takuma Y. [Helicobacter pylori infection and liver diseases]. Gan to Kagaku Ryoho. 2011; 38(3):362-4.

26. Polyzos SA, Kountouras J, Papatheodorou A, Patsiaoura K, Katsiki E, Zafeiriadou E, et al. Helicobacter pylori infection in patients with nonalcoholic fatty liver disease. Metabolism. 2013; 62(1):121-6.

27. Aller R, De Luis DA, Izaola O, Conde R, Gonzalez Sagrado M, Primo D, et al. Effect of a probiotic on liver aminotransferases in nonalcoholic fatty liver disease patients: a double blind randomized clinical trial. Eur Rev Med Pharmacol Sci. 2011; 15(9):1090-5.

28. Solga SF, Diehl AM. Non-alcoholic fatty liver disease: lumen-liver interactions and possible role for probiotics. J Hepatol. 2003; 38(5):681-7.

29. Promrat K, Kleiner DE, Niemeier HM, Jackvony E, Kearns M, Wands JR, et al. Randomized controlled trial testing the effects of weight loss on nonalcoholic steatohepatitis. Hepatology. 2010; 51(1):121-9.

30. Dumas ME, Barton RH, Toye A, Cloarec O, Blancher C, Rothwell A, et al. Metabolic profiling reveals a contribution of gut microbiota to fatty liver phenotype in insulin-resistant mice. Proc Natl Acad Sci U S A. 2006; 103(33):12511-6. 